PROCEEDINGS OF THE

AMERICAN MATHEMATICAL SOCIETY

Volume 133, Number 5, Pages 1307-1315

S 0002-9939(04)07685-3

Article electronically published on November 1, 2004

\title{
MORE ON CONVEXITY NUMBERS OF CLOSED SETS IN $\mathbb{R}^{n}$
}

\author{
STEFAN GESCHKE
}

(Communicated by Carl G. Jockusch, Jr.)

\begin{abstract}
The convexity number of a set $S \subseteq \mathbb{R}^{n}$ is the least size of a family $\mathcal{F}$ of convex sets with $\bigcup \mathcal{F}=S . S$ is countably convex if its convexity number is countable. Otherwise $S$ is uncountably convex.

Uncountably convex closed sets in $\mathbb{R}^{n}$ have been studied recently by Geschke, Kubiś, Kojman and Schipperus. Their line of research is continued in the present article. We show that for all $n \geq 2$, it is consistent that there is an uncountably convex closed set $S \subseteq \mathbb{R}^{n+1}$ whose convexity number is strictly smaller than all convexity numbers of uncountably convex subsets of $\mathbb{R}^{n}$.

Moreover, we construct a closed set $S \subseteq \mathbb{R}^{3}$ whose convexity number is $2^{\aleph_{0}}$ and that has no uncountable $k$-clique for any $k>1$. Here $C \subseteq S$ is a $k$-clique if the convex hull of no $k$-element subset of $C$ is included in $S$. Our example shows that the main result of the above-named authors, a closed set $S \subseteq \mathbb{R}^{2}$ either has a perfect 3-clique or the convexity number of $S$ is $<2^{\aleph_{0}}$ in some forcing extension of the universe, cannot be extended to higher dimensions.
\end{abstract}

\section{INTRODUCTION}

There is a natural way to quantify the non-convexity of a subset $S$ of a real vector space. $S$ is close to being convex if its convexity number $\gamma(S)$, the least size of a family of convex subsets of $S$ that covers $S$, is small. We are mainly interested in uncountably convex sets, that is, sets whose convexity number is uncountable. One obvious reason for an uncountable convexity number is the existence of an uncountable $k$-clique for some $k>1$ where a set $C \subseteq S$ is a $k$-clique if for all $F \in[C]^{k}$ the convex hull $\operatorname{conv}(F)$ is not included in $S$.

If we restrict our attention to closed subsets of Polish real vector spaces, then the existence of an uncountable $k$-clique for some $k>1$ implies that there is a perfect $k$-clique [7]. In particular, if $X$ is a Polish real vector space and $S \subseteq X$ is closed and has an uncountable $k$-clique for some $k>1$, then $\gamma(S)=2^{\aleph_{0}}$. Therefore, it is natural to ask whether it is consistent for $n>0$ that there is a closed set $S \subseteq \mathbb{R}^{n}$ with $\aleph_{0}<\gamma(S)<2^{\aleph_{0}}$.

Recall Carathéodory's theorem: The convex hull of a set $X \subseteq \mathbb{R}^{n}$ is the union of the convex hulls of its $(n+1)$-element subsets. This implies that every infinite

Received by the editors August 18, 2003 and, in revised form, December 1, 2003 and January 16, 2004.

2000 Mathematics Subject Classification. Primary 05A20, 52A05, 03E17, 03E35; Secondary $03 \mathrm{E} 75$.

Key words and phrases. Convex cover, convexity number, clique, $n$-space, forcing extension, covering number. 
$k$-clique of a set $S \subseteq \mathbb{R}^{n}$ includes an $(n+1)$-clique of the same size. It follows that if we are interested in the convexity structure of subsets of $\mathbb{R}^{n}$, we should consider $(n+1)$-cliques.

It is easily checked that every uncountably convex, closed subset of $\mathbb{R}$ has a perfect 2-clique. The analogue for the plane, namely that every uncountably convex closed subset of $\mathbb{R}^{2}$ has a perfect 3 -clique, was supposedly proved in [6] but turned out to be wrong. In [2 it was shown that there is an uncountably convex, closed subset of the plane whose convexity number is consistently $<2^{\aleph_{0}}$.

For $\mathbb{R}^{3}$ the existence of such a set has been established earlier in [6] (see [5] for an improved construction). Both constructions have in common that they can be carried out in ZFC. Then forcing has to be used to construct models of set theory where the respective convexity numbers are small. The construction of the $\mathbb{R}^{3}$-example has been generalized in [3] in order to show that for every $n \geq 3$ it is consistent that

$$
\sigma\left(\mathbb{R}^{n}\right):=\left\{\gamma(S): S \subseteq \mathbb{R}^{n} \text { is closed and uncountably convex }\right\},
$$

the convexity spectrum of $\mathbb{R}^{n}$, is of size $\geq n-1$.

This result indicates (but does not prove) that for every $n \geq 3$ the convexity spectrum of $\mathbb{R}^{n+1}$ can contain more cardinals than the convexity spectrum of $\mathbb{R}^{n}$. In Section 2 we show that for every $n \geq 2$ it is consistent that there is a closed subset of $\mathbb{R}^{n+1}$ whose convexity number is uncountable and strictly smaller than all uncountable convexity numbers of closed subsets of $\mathbb{R}^{n}$.

Note the following: If $S \subseteq \mathbb{R}^{n}$ is Borel and has a perfect $k$-clique for some $k>1$, then it has a perfect $k$-clique in every forcing extension of the universe. In particular, if $S$ is closed and $\gamma(S)<2^{\aleph_{0}}$ holds in some forcing extension of the universe, then $S$ has no uncountable cliques. This implication can be reversed for $n=2$. In 2 it was shown that adding $\left(2^{\aleph_{0}}\right)+$ Sacks reals to the universe using countable support iteration yields a model of set theory where $\gamma(S)<2^{\aleph_{0}}$ for every closed set $S \subseteq \mathbb{R}^{2}$ that has no uncountable 3-cliques.

In Section 3 we show that the analogue in dimension 3 fails: there is a closed set $S \subseteq \mathbb{R}^{3}$ without uncountable cliques whose convexity number is $2^{\aleph_{0}}$ in ZFC.

\section{CONVEXity NUmbers IN $\mathbb{R}^{n}$ VERsus $\mathbb{R}^{n+1}$}

We use a reformulation of convexity numbers as chromatic numbers of certain hypergraphs that was observed in [5].

Let $S$ be a subset of a real vector space. A set $F \subseteq S$ is defected in $S$ if $\operatorname{conv}(F)$ is not included in $S$. Consider the hypergraph $(S, E)$ where $E$ consists of all finite defected subsets of $S$. The chromatic number of $(S, E)$, i.e., the least size of a family $\mathcal{F} \subseteq \mathcal{P}(S)$ such that $S=\bigcup \mathcal{F}$ and $\mathcal{P}(F) \cap E=\emptyset$ for every $F \in \mathcal{F}$, is precisely $\gamma(S)$. This is because a subset of $S$ is defected if and only if one of its finite subsets is defected. A set $F \subseteq S$ with $\mathcal{P}(F) \cap E=\emptyset$ is an independent set. The cliques in $S$ with respect to convexity are precisely the cliques of $(S, E)$ in the graph-theoretic sense: a set $C \subseteq S$ is a $k$-clique of $(S, E)$ if $[C]^{k} \subseteq E$.

If $S$ is a subset of $\mathbb{R}^{n}$, then, again by Carathéodory's theorem, it is enough to study the defected subsets of $S$ of size $n+1$. Therefore, we define the defectedness hypergraph of $S$ to be the structure $(S, E)$ where

$$
E:=\left\{F \in[S]^{n+1}: F \text { is defected in } S\right\} .
$$


Still the chromatic number of $(S, E)$ is $\gamma(S)$. $(S, E)$ is what is called an $(n+1)$ regular hypergraph, i.e., a hypergraph whose edges are all of size $n+1$. If $S$ is closed, then $E$ is an open subset of $[S]^{n+1}$ with respect to the topology inherited from the product topology on $S^{n+1}$, i.e., the hypergraph is open.

For $n \geq 2$ let $I_{n}$ be the $\sigma$-ideal on $n^{\omega}$ generated by those closed sets that do not contain more than $n-1$ equidistant points with respect to the usual metric on $n^{\omega}$. In other words, the generators of $I_{n}$ correspond to subtrees of $n^{<\omega}$ in which no node has more than $n-1$ immediate successors. For an ideal $I$ on a space $X$ let $\operatorname{cov}(I)$ denote the covering number of $I$, i.e., the least size of a family $\mathcal{F} \subseteq I$ such that $X=\bigcup \mathcal{F}$.

In 6] the uncountable convexity of a closed set $S \subseteq \mathbb{R}^{n}$ has been characterized as follows: $S$ is uncountably convex if and only if there is a continuous embedding $e:(n+1)^{\omega} \rightarrow S$ such that for every set $F \in\left[(n+1)^{\omega}\right]^{n+1}$ consisting of equidistant points, $e[F]$ is defected in $S$. This immediately shows

Theorem 2.1. Let $n \geq 1$. Let $S \subseteq \mathbb{R}^{n}$ be a closed set that is not countably convex. Then the convexity number of $S$ is at least $\operatorname{cov}\left(I_{n+1}\right)$.

In [3] for every $n \geq 2$ a closed subset of $\mathbb{R}^{n}$ has been constructed whose convexity number is $\operatorname{cov}\left(I_{n}\right)$. In the view of Theorem 2.1 it is tempting to try to construct a set in $\mathbb{R}^{n}$ whose convexity number is $\operatorname{cov}\left(I_{n+1}\right)$. We show that this cannot be done in ZFC. More precisely, we show that every model of set theory has a forcing extension in which all convexity numbers of closed uncountably convex subsets of $\mathbb{R}^{n}$ are strictly larger than $\operatorname{cov}\left(I_{n+1}\right)$.

One geometric ingredient for this is still missing: the transitivity of the defectedness hypergraph.

Definition 2.2. Let $n \geq 2, V$ a set, and $E \subseteq[V]^{n}$. Then the hypergraph $(V, E)$ is transitive if for all $e \in E$ and all $v \in V \backslash e$ there is $w \in e$ such that $(e \backslash\{w\}) \cup\{v\} \in$ E.

Note that all induced subgraphs of a transitive, $n$-regular hypergraph are transitive.

Lemma 2.3. For all $S \subseteq \mathbb{R}^{n}$ the defectedness hypergraph of $S$ is transitive.

Proof. Suppose $\left\{x_{0}, \ldots, x_{n}\right\} \in[S]^{n+1}$ is defected. Let $z \in \operatorname{conv}\left(\left\{x_{0}, \ldots, x_{n}\right\}\right) \backslash S$. For all $y \in S \backslash\left\{x_{0}, \ldots, x_{n}\right\}$ at least one of the sets $\operatorname{conv}(\{y\} \cup e), e \in\left[\left\{x_{0}, \ldots, x_{n}\right\}\right]^{n}$, contains $z$ and therefore is defected in $S$.

A hypergraph $(V, E)$ is uncountably chromatic if its chromatic number is uncountable. For $A \subseteq V,(V, E)$ is uncountably chromatic on $A$ if $\left(A, E \cap[A]^{k}\right)$ is uncountably chromatic.

Theorem 2.4. For every $n \geq 2$ the following is consistent with ZFC:

For every Polish space $V$ and every $E \subseteq[V]^{n+1}$, if $(V, E)$ is an uncountably chromatic, transitive, open hypergraph, then the chromatic number of $(V, E)$ is $\aleph_{2}$ while $\operatorname{cov}\left(I_{n+1}\right)=\aleph_{1}$.

Before proving this theorem, we derive from it

Corollary 2.5. For all $n \geq 2$ there is an uncountably convex, closed set $S \subseteq \mathbb{R}^{n+1}$ whose convexity number is consistently smaller than any convexity number of an uncountably convex, closed subset of $\mathbb{R}^{n}$. 
Proof. Let $S \subseteq \mathbb{R}^{n+1}$ be a closed set with $\gamma(S)=\operatorname{cov}\left(I_{n+1}\right)$. The existence of such a set was shown in [3], as mentioned above. Now the corollary follows from Theorem 2.1. Lemma 2.3 Theorem 2.4 and the characterization of convexity numbers as chromatic numbers of defectedness hypergraphs.

The proof of Theorem 2.4 uses a countable support iteration of length $\omega_{2}$ over a model of $\mathrm{CH}$ (or, to be more general, a countable support iteration of length $\left(2^{\aleph_{0}}\right)^{+}$over any model of set theory). For the successor steps of the iteration we need a forcing notion that increases the chromatic number of a given uncountably chromatic, $(n+1)$-regular hypergraph $G=(V, E)$ but keeps $\operatorname{cov}\left(I_{n+1}\right)$ small. The most natural approach works:

Definition 2.6. For every uncountably chromatic, $(n+1)$-regular open hypergraph $G=(V, E)$ on a Polish space $V$, let $\mathbb{P}_{G}$ be the set of closed sets $C \subseteq V$ such that the induced subgraph on $C$ is uncountably chromatic on every non-empty open subset of $C$. The order on $\mathbb{P}_{G}$ is set-theoretic inclusion.

First observe that $\mathbb{P}_{G}$ is non-empty. In fact, it has a largest element. The largest element of $\mathbb{P}_{G}$ is obtained by iteratively removing all open subsets from $V$ on which the graph is countably chromatic. The procedure terminates after countably many steps since $V$ is second countable. Finally, we are left with a subset of $V$ that is a condition in $\mathbb{P}_{G}$. If $G$ is the defectedness hypergraph of an uncountably convex, closed set $S \subseteq \mathbb{R}^{n}$, then the largest element of $\mathbb{P}_{G}$ is the so-called convexity radical of $S$ as introduced in [5].

It is easily checked that $\mathbb{P}_{G}$ adds an element of $V$ that is not contained in any independent subset of $V$ that is coded in the ground model. Starting from a model of $\mathrm{CH}$ and using some bookkeeping we can construct a countable support iteration of length $\omega_{2}$ of forcing notions of the type $\mathbb{P}_{G}$ such that for every relevant hypergraph $G$ in the final model, the forcing notion $\mathbb{P}_{G}$ appears cofinally often in the iteration, making sure that the chromatic number of $G$ in the final model is $\aleph_{2}$. This can be done since every relevant hypergraph can be coded by a single real and therefore is added in some initial stage of the iteration. We have to show that such an iteration does not increase $\operatorname{cov}\left(I_{n+1}\right)$. This is where transitivity comes in.

Definition 2.7. A forcing notion $\mathbb{P}$ has the $k$-localization property for $k>1$ if for every $\mathbb{P}$-name $\dot{x}$ for an element of $\omega^{\omega}$ it is forced that $\dot{x}$ is a branch of a subtree $T$ of $\omega^{<\omega}$ such that $T$ is a element of the ground model and every element of $T$ has at most $k$ immediate successors in $T$ (i.e., $T$ is $k$-ary).

In particular, if $\mathbb{P}$ has the $n$-localization property, then every new element of $(n+1)^{\omega}$ added by $\mathbb{P}$ is contained in a ground model element of $I_{n+1}$.

Lemma 2.8. If $G=(V, E)$ is an uncountably chromatic, open, transitive, $(n+1)$ regular hypergraph on the Polish space $V$ such that $G$ is uncountably chromatic on every open subset of $V$, then the forcing notion $\mathbb{P}_{G}$ has the $n$-localization property.

Proof. Let $\dot{x}$ be a $\mathbb{P}_{G}$-name for an element of $\omega^{\omega}$ that is not in the ground model. For every condition $p \in \mathbb{P}_{G}$ we have to find $q \leq p$ such that

$$
T_{q}(\dot{x}):=\left\{t \in \omega^{<\omega}: \exists r \leq q(r \Vdash t \subseteq \dot{x})\right\},
$$

the tree of $q$-possibilities for $\dot{x}$, is $n$-ary. 
For every condition $r \in \mathbb{P}_{G}$ let $\dot{x}[r]$ be the longest initial segment of $\dot{x}$ that is decided by $r$. Since $\dot{x}$ is a name for a new real, $\dot{x}[r]$ is finite. For $\sigma, \tau \in \omega^{<\omega}$ we write $\sigma \perp \tau$ if $\sigma \nsubseteq \tau$ and $\tau \nsubseteq \sigma$.

Fix $p \in \mathbb{P}_{G}$. Let $I$ be the set of all $\sigma \in n^{<\omega}$ such that for all $k \in \omega$, if $2 k \in \operatorname{dom}(\sigma)$, then $\sigma(2 k) \in 2$ and moreover, if $\sigma(2 k)=0$ and $2 k+1 \in \operatorname{dom}(\sigma)$, then $\sigma(2 k+1)=0$. We will construct a family $\left(q_{\sigma}\right)_{\sigma \in I}$ of conditions such that

(i) $q_{\emptyset}=p$,

(ii) for all $\sigma, \tau \in I$, if $\sigma \perp \tau$, then $q_{\sigma} \cap q_{\tau}=\emptyset$ and $\dot{x}\left[q_{\sigma}\right] \perp \dot{x}\left[q_{\tau}\right]$,

(iii) for all $\sigma \in I$, the diameter of $q_{\sigma}$ is at most $2^{-|\sigma|}$, and

(iv) for all $k \in \omega$ and all $\sigma \in n^{2 k}$, if $v \in q_{\sigma \frown 0 \frown 0}$ and for all $i<n, w_{i} \in q_{\sigma \frown 1 \frown i}$, then $\left\{v, w_{0}, \ldots, w_{n-1}\right\} \in E$.

Suppose $\left(q_{\sigma}\right)_{\sigma \in I}$ is a family of conditions below $p$ satisfying (i)-(iv). Let $q:=$ $\bigcap_{k \in \omega} \bigcup\left\{q_{\sigma}: \sigma \in I \cap n^{k}\right\}$. Then $q$ is a closed subset of $p$ by (i). By (iii) and (iv), $q$ is non-empty and the induced subgraph on $q$ is uncountably chromatic on every open subset of $q$. Therefore, $q \in \mathbb{P}_{G}$ and $q \leq p$. It follows from (ii) that $T_{q}(\dot{x})$ is $n$-ary.

It remains to construct a family $\left(q_{\sigma}\right)_{\sigma \in I}$ with the required properties. Suppose we have constructed $q_{\sigma}$ for some $\sigma \in I$ with an even domain. Let $q_{\sigma \frown 0}, q_{\sigma \frown 1} \leq$ $q_{\sigma}$ be disjoint and such that $\dot{x}\left[q_{\sigma-0}\right] \perp \dot{x}\left[q_{\sigma-1}\right]$. This is possible since $\dot{x}$ is a name for a new element of $\omega^{\omega}$. Since $G$ is uncountably chromatic on $q_{\sigma \frown 1}$, there are $v_{0}, \ldots, v_{n} \in q_{\sigma \frown 1}$ such that $\left\{v_{0}, \ldots, v_{n}\right\} \in E$. Choose $w \in q_{\sigma \frown 0}$. By the transitivity of $G$, there is $e \in\left[\left\{v_{0}, \ldots, v_{n}\right\}\right]^{n}$ such that $e \cup\{w\} \in E$. Without loss of generality, $e=\left\{v_{0}, \ldots, v_{n-1}\right\}$. Since $E$ is open, there are pairwise disjoint open sets $W, U_{0}, \ldots, U_{n-1} \subseteq V$ such that $w \in W, v_{i} \in U_{i}$ for all $i<n$, and for all $w^{\prime} \in W$ and $v_{i}^{\prime} \in U_{i}, i<n$, we have $\left\{w^{\prime}, v_{0}^{\prime}, \ldots, v_{n-1}^{\prime}\right\} \in E$.

We can now choose $q_{\sigma \frown 0 \frown 0} \leq q_{\sigma \frown 0} \cap W$ and $q_{\sigma \frown 1 \frown i} \leq q_{\sigma \frown 1} \cap U_{i}$ for all $i<n$ such that (ii) and (iii) are satisfied. The choice of $W$ and of the $U_{i}$ guarantees (iv).

Using the usual fusion technology for Axiom A forcings and the argument in the proof of Lemma 2.8 it is easily checked that countable support iterations of forcing notions of the type $\mathbb{P}_{G}, G$ an open, transitive, uncountably chromatic, $(n+1)$ regular hypergraph on a Polish space, have the $n$-localization property. The proof is in fact a simple generalization of the proof that countable support iterations of Sacks forcing have the 2-localization property (see [1]). This finishes the proof of Theorem 2.4.

\section{A COUnTEREXAMPLe in $\mathbb{R}^{3}$}

As mentioned in the introduction, it was shown in 2] that for every closed subset $S$ of the plane exactly one of the following statements is true:

(i) $S$ includes a perfect 3 -clique.

(ii) In some forcing extension of the universe, $\gamma(S)<2^{\aleph_{0}}$.

It is reasonable to conjecture that this dichotomy has a higher-dimensional analogue. However, as we show in this section, this is not the case. There is a closed subset $S$ of $\mathbb{R}^{3}$ such that $S$ has no uncountable 3 -clique, but the convexity number of $S$ is $2^{\aleph_{0}}$ (in ZFC).

The construction of $S$ uses the following coloring $c$ of a 3 -element subset of $2^{\omega}$. 
Definition 3.1. For $\{x, y\} \in\left[2^{\omega}\right]^{2}$ let $\Delta(x, y)$ denote the first $n \in \omega$ such that $x(n) \neq y(n)$. Let $\leq_{\text {lex }}$ denote the lexicographic order on $2^{\omega}$. For $\{x, y, z\} \in\left[2^{\omega}\right]^{3}$ with $x \leq_{\operatorname{lex}} y \leq_{\operatorname{lex}} z$, let $c(x, y, z):=0$ if and only if $\Delta(x, y)<\Delta(y, z)$ and $c(x, y, z):=1$ otherwise. Note that $\Delta(x, y) \neq \Delta(y, z)$.

If $\rho, \sigma, \tau \in 2^{<\omega}$ are pairwise incomparable (with respect to $\subseteq$ ), let $\bar{c}(\rho, \sigma, \tau):=$ $c(x, y, z)$ where $x, y, z \in 2^{\omega}$ are chosen such that $\rho \subseteq x, \sigma \subseteq y$, and $\tau \subseteq z$. Since $\rho, \sigma, \tau$ are pairwise incomparable, $\bar{c}(\rho, \sigma, \tau)$ is well-defined. Similarly, if $\sigma, \tau \in 2^{\omega}$ are incomparable, then we write $\sigma \leq_{\operatorname{lex}} \tau$ if and only if for all $x, y \in 2^{\omega}$ with $\sigma \subseteq x$ and $\tau \subseteq y$ we have $x \leq_{\text {lex }} y$.

A set $H \subseteq 2^{\omega}$ is homogeneous (with respect to $c$ ) if $c$ is constant on $[H]^{3}$.

The coloring $c$ is clearly continuous, i.e., $c^{-1}(0)$ and $c^{-1}(1)$ are open subsets of $\left[2^{\omega}\right]^{3}$. While for continuous colorings of 2 -element subsets of $2^{\omega}$ we always have perfect homogeneous sets by a theorem of Galvin (see [4, Theorem 19.7]), this is not the case for $c$. The following lemma seems to be well known (see [4, Exercise 19.10]).

Lemma 3.2. Let $H \subseteq 2^{\omega}$ be c-homogeneous. Then $H$ is at most countable.

The connection between the coloring $c$ and convex geometry in $\mathbb{R}^{3}$ is as follows:

Lemma 3.3. There are a closed set $S \subseteq \mathbb{R}^{3}$ and an embedding $e: 2^{\omega} \rightarrow S$ such that $e\left[2^{\omega}\right]$ is the convexity radical of $S$ and for all $X \subseteq 2^{\omega}$ the convex hull of $e[X]$ is included in $S$ if and only if $X$ is c-homogeneous of color 1.

Proof. $S$ will be obtained by removing countably many open cuboids from $\mathbb{R}^{3}$. The point in removing open cuboids instead of open balls or similar objects is that the complement of an open cuboid in $\mathbb{R}^{3}$ is a closed set that is countably convex while the complement of an open ball includes a perfect 2-clique, namely the boundary of the ball.

Let us fix some notation first. Let $I:=(0,1)$ and $f: I \rightarrow \mathbb{R}^{3} ; t \mapsto(\sin t, \cos t, t)$. For $t \in(0,1)$ let $P_{t}$ denote the plane through $(0,0,0)$ that is orthogonal to the vector $(\sin t, \cos t, 0)$, and let $p_{t}: \mathbb{R}^{3} \rightarrow P_{t}$ denote the orthogonal projection onto $P_{t}$. Finally, let

$$
q: \mathbb{R}^{3} \rightarrow \mathbb{R}^{2} ;\left(x_{0}, x_{1}, x_{2}\right) \mapsto\left(x_{0}, x_{1}\right) .
$$

For every $\sigma \in 2^{<\omega}$ we choose $t_{\sigma} \in I$, an open set $O_{\sigma} \subseteq I$, and an open cuboid $C_{\sigma} \subseteq \mathbb{R}^{3}$ such that the following conditions are satisfied:

(1) For every $\sigma \in 2^{<\omega}, O_{\sigma}$ is an open interval centered at $t_{\sigma}$.

(2) For every $n$ and every $\sigma \in 2^{n}$ the diameter of $O_{\sigma}$ is at most $2^{-n}$.

(3) For all $\sigma, \tau \in 2^{<\omega}, \sigma \varsubsetneqq \tau$ implies $\operatorname{cl}\left(O_{\tau}\right) \subseteq O_{\sigma}$.

(4) If $\sigma, \tau \in 2^{<\omega}$ are incomparable, then $\operatorname{cl}\left(O_{\sigma}\right) \cap \operatorname{cl}\left(O_{\tau}\right)=\emptyset$. If additionally $\sigma \leq_{\text {lex }} \tau$, then $t_{\sigma} \leq t_{\tau}$.

(5) For all $\sigma \in 2^{<\omega}$,

$$
q\left[\operatorname{conv}\left(f\left[O_{\sigma \frown 0} \cup O_{\sigma \frown 1}\right]\right)\right] \cap q\left[C_{\sigma}\right]=\emptyset .
$$

(6) If $\sigma \in 2^{<\omega}$ is incomparable with $\tau_{0}, \tau_{1} \in 2^{<\omega}$, then for all $i \in 2$,

$$
q\left[\operatorname{conv}\left(f\left[O_{\sigma-i} \cup O_{\tau_{0}} \cup O_{\tau_{1}}\right]\right)\right] \cap q\left[C_{\sigma}\right]=\emptyset .
$$

(7) If $\sigma, \tau \in 2^{<\omega}$ are incomparable and $\tau \leq_{\text {lex }} \sigma$, then for all $x_{0} \in f\left[O_{\sigma-0}\right]$, $x_{1} \in f\left[O_{\sigma \frown 1}\right]$, and $y \in f\left[O_{\tau}\right]$ it is the case that

$$
C_{\sigma} \cap \operatorname{conv}\left(x_{0}, x_{1}, y\right) \neq \emptyset \text {. }
$$




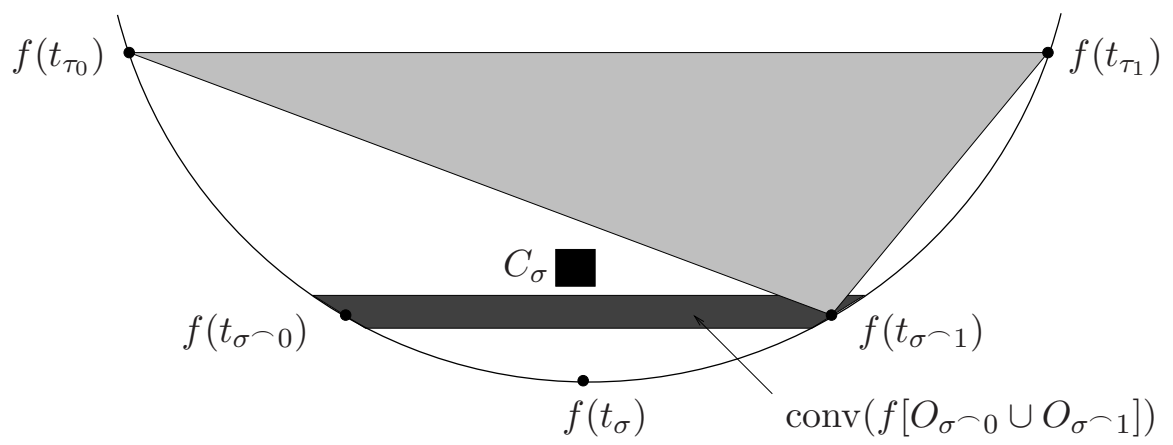

Figure 1. Illustration of the conditions (5) and (6). Projection onto the plane via $q$. For simplicity, the sets $f\left[O_{\eta}\right], \eta \in\left\{\sigma, \tau_{0}, \tau_{1}\right\}$, are hidden.

(8) If $\sigma \in 2^{<\omega}$ is incomparable with $\tau_{0}, \tau_{1} \in 2^{<\omega}$ and $\sigma \leq_{\operatorname{lex}} \tau_{0}, \tau_{1}$, then

$$
p_{t_{\sigma}}\left[\operatorname{conv}\left(f\left[O_{\sigma \frown 0} \cup O_{\sigma-1} \cup O_{\tau_{0}} \cup O_{\tau_{1}}\right]\right)\right] \cap p_{t_{\sigma}}\left[C_{\sigma}\right]=\emptyset .
$$

(9) For all $n \in \omega$ and all $\sigma \in 2^{n}, C_{\sigma}$ is included in the ball of diameter $2^{1-n}$ centered at $f\left(t_{\sigma}\right)$.

This construction can be carried out: (1)-(4) are easy to satisfy. For (5) and (6) we choose the projection $q\left[C_{\sigma}\right]$ first. The two conditions can be satisfied since $q[f[I]]$ lies on a circle. If we choose $q\left[C_{\sigma}\right]$ as indicated in Figure 1, we will not have problems satisfying the remaining conditions.

For (7) and (8) consider the projection $p_{t_{\sigma}}[f[I]]$ of $f[I]$ to the plane $P_{t_{\sigma}}$. The set $p_{t_{\sigma}}[f[I]]$ is a part of the graph of the arcsin-function (identifying $P_{t_{\sigma}}$ with $\mathbb{R}^{2}$ ). By adjusting the bottom and the top of $C_{\sigma}$ correctly, (7) and (8) can be satisfied simultaneously since arcsin is strictly concave on negative arguments and strictly convex on positive arguments.

Taking care of (9) in the inductive construction is easy since $O_{\sigma}$ is sufficiently small and the height of $C_{\sigma}$ can be chosen smaller as $q\left[C_{\sigma}\right]$ gets closer to $q\left(f\left(t_{\sigma}\right)\right)$.

Now let $\left(t_{\sigma}, O_{\sigma}, C_{\sigma}\right)_{\sigma \in 2}<\omega$ be such that (1)-(9) are satisfied. For each $x \in 2^{\omega}$, let $e(x):=f\left(\lim _{n \rightarrow \infty} t_{x \uparrow n}\right)$. (1)-(4) guarantee that $e$ is a continuous embedding. Let $S:=\mathbb{R}^{3} \backslash \bigcup_{\sigma \in 2<\omega} C_{\sigma}$. By (9), every point in $\mathbb{R}^{3} \backslash e\left[2^{\omega}\right]$ has a neighborhood that intersects only finitely many of the $C_{\sigma}$. It follows that the convexity radical of $S$ is the set $e\left[2^{\omega}\right]$.

For every $X \subseteq 2^{\omega}$ we have the following: If $X$ has a 3 -element subset $\left\{x_{0}, x_{1}, x_{2}\right\}$ such that $x_{0} \leq_{\text {lex }} x_{1} \leq_{\text {lex }} x_{2}$ and $\Delta\left(x_{0}, x_{1}\right)<\Delta\left(x_{1}, x_{2}\right)$, then by $(7)$, for $\sigma:=x_{1} \uparrow$ $\Delta\left(x_{1}, x_{2}\right)$ we have

$$
\operatorname{conv}\left(e\left(x_{0}\right), e\left(x_{1}\right), e\left(x_{2}\right)\right) \cap C_{\sigma} \neq \emptyset .
$$

In particular, $\operatorname{conv}(e[X]) \nsubseteq \subseteq$. It follows that every $X \subseteq 2^{\omega}$ with $\operatorname{conv}(e[X]) \subseteq S$ is $c$-homogeneous of color 1 .

Now suppose that $X \subseteq 2^{\omega}$ is $c$-homogeneous of color 1 . Then $\operatorname{conv}(e[X]) \subseteq S$ provided that no 4 -element subset of $e[X]$ is defected in $S$. Therefore, without loss of generality, we may assume that $X$ itself is of size 4 , say $X=\left\{x_{0}, x_{1}, x_{2}, x_{3}\right\}$ with $x_{0} \leq_{\text {lex }} x_{1} \leq_{\text {lex }} x_{2} \leq_{\text {lex }} x_{3}$. Let $\sigma \in 2^{<\omega}$. We have to show that $C_{\sigma}$ is disjoint from $\operatorname{conv}(e[X])$. 


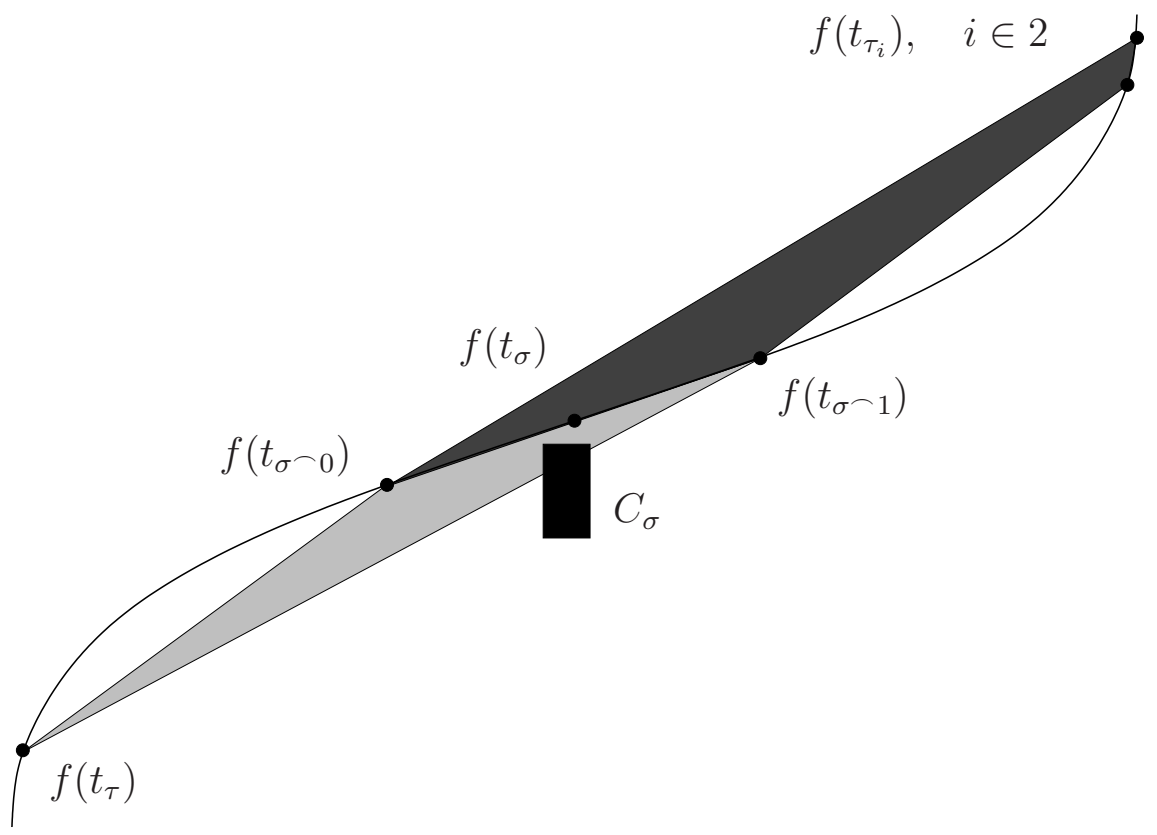

FiguRe 2. Illustration of the conditions (7) and (8). Projection onto the plane via $p_{t_{\sigma}}$. The sets $f\left[O_{\eta}\right], \eta \in\left\{\sigma \frown 0, \sigma \frown 1, \tau, \tau_{0}, \tau_{1}\right\}$, are hidden.

First consider the projection to $\mathbb{R}^{2}$ via $q$. Suppose $q\left[C_{\sigma}\right]$ intersects $q[\operatorname{conv}(e[X])]$. Then $q\left[C_{\sigma}\right]$ intersects the convex hull of a 3-element subset of $(q \circ e)[X]$, say of $(q \circ e)\left[\left\{y_{0}, y_{1}, y_{2}\right\}\right]$, where $y_{0}, y_{1}, y_{2} \in X$.

Claim 3.4. For every $i \in 2, \sigma^{\frown} i$ is comparable with exactly one element of $\left\{y_{0}, y_{1}, y_{2}\right\}$.

Suppose $\sigma^{\frown} i$ is incomparable with $y_{0}, y_{1}$, and $y_{2}$. Then there are $\tau_{0}, \tau_{1} \in 2^{<\omega}$, incomparable with $\sigma$, such that

$$
e\left[\left\{y_{0}, y_{1}, y_{2}\right\}\right] \subseteq f\left[O_{\sigma} \frown_{i} \cup O_{\tau_{0}} \cup O_{\tau_{1}}\right] .
$$

Note that $\tau_{0}$ or $\tau_{1}$ (or both) might be unneccessary here, for instance if more than one element of $\left\{y_{0}, y_{1}, y_{2}\right\}$ is comparable with $\sigma^{-}(1-i)$. By the choice of $\left\{y_{0}, y_{1}, y_{2}\right\}$,

$$
q\left[C_{\sigma}\right] \cap q\left[\operatorname{conv}\left(f\left[O_{\sigma \frown i} \cup O_{\tau_{0}} \cup O_{\tau_{1}}\right]\right)\right] \neq \emptyset,
$$

but this contradicts $(6)$.

It follows that $\sigma^{\frown} 0$ and $\sigma^{\frown 1}$ are each comparable with at least one element of $\left\{y_{0}, y_{1}, y_{2}\right\}$.

If $\sigma^{\frown} i$ is comparable with more than one element of $\left\{y_{0}, y_{1}, y_{2}\right\}$, then $y_{0}, y_{1}$, and $y_{2}$ are all comparable with $\sigma$. In particular,

$$
e\left[\left\{y_{0}, y_{1}, y_{2}\right\}\right] \subseteq f\left[O_{\sigma \frown 0} \cup O_{\sigma-1}\right]
$$

and thus

$$
q\left[C_{\sigma}\right] \cap q\left[\operatorname{conv}\left(f\left[O_{\sigma \frown 0} \cup O_{\sigma-1}\right]\right)\right] \neq \emptyset,
$$

contradicting (5). This shows the claim. 
Since $X$ is $c$-homogeneous of color 1 , the claim implies that either $\sigma \frown 0 \subseteq x_{0}$, $\sigma^{\frown} 1 \subseteq x_{1}$, and $\sigma \nsubseteq x_{2}, x_{3}$ or $\sigma \frown 0 \subseteq x_{0}, x_{1}, \sigma \frown 1 \subseteq x_{2}$, and $\sigma \nsubseteq x_{3}$. In both cases it follows from (8) that $p_{t_{\sigma}}[\operatorname{conv}(e[X])]$ is disjoint from $p_{t_{\sigma}}\left[C_{\sigma}\right]$. All in all we get that $C_{\sigma}$ is disjoint from $\operatorname{conv}(e[X])$. Since $\sigma$ was arbitrary, we have $\operatorname{conv}(e[X]) \subseteq S$.

Note that Lemma 3.3 shows that there is an uncountably convex, closed set $S \subseteq \mathbb{R}^{3}$ such that the $\sigma$-ideal generated by the convex subsets of $S$ restricted to the convexity radical of $S$ is precisely the ideal of countable subsets of the convexity radical.

Lemma 3.2 and Lemma 3.3 easily imply

Corollary 3.5. There is a closed set $S \subseteq \mathbb{R}^{3}$ without uncountable $n$-cliques for any $n \in \omega \backslash 2$ whose convexity number is $2^{\aleph_{0}}$.

\section{REFERENCES}

[1] S. Geschke, S. Quickert, On Sacks forcing and the Sacks property, accepted for publication in Foundations of the Formal Sciences II (B. Löwe, W. Malzkorn, T. Räsch eds.), Applications of Mathematical Logic in Philosophy and Linguistics, Bonn, November 10-13, 2000

[2] S. Geschke, W. Kubiś, M. Kojman, R. Schipperus, Convex decompositions in the plane, meagre ideals and continuous pair colorings of the irrationals, Israel Journal of Mathematics 131, 285-317 (2002) MR1942314 (2004f:52001)

[3] S. Geschke, M. Kojman, Convexity numbers of closed subsets in $\mathbb{R}^{n}$, Proc. Am. Math. Soc. 130, No.10, 2871-2881 (2002) MR1908910 (2003e:03093)

[4] A. Kechris, Classical Descriptive Set Theory, Graduate Texts in Mathematics, Springer (1995) MR.1321597 (96e:03057)

[5] M. Kojman, Degrees of non convexity in higher dimension, Fundamenta Mathematicae (164), 143-163 (2000) MR1784705 (2001g:52003)

[6] M. Kojman, M. Perles, S. Shelah, Sets in a Euclidean space which are not a countable union of convex subsets, Isreal Journal of Mathematics 70 (3) (1990), 313-342 MR.1074495 (92e:52006)

[7] W. Kubiś, Perfect cliques and $G_{\delta}$ colorings of Polish spaces, Proc. Amer. Math. Soc. 131 (2003), no. 2, 619-623 (electronic) MR.1933354 (2004g:54043)

II. Mathematisches Institut, Freie Universität Berlin, Arnimallee 3, 14195 Berlin, Germany

E-mail address: geschke@math.fu-berlin.de 